\title{
Potassium and Ion Beam induced Electron Accumulation in InN
}

\author{
L. Colakerol, ${ }^{1,2}$ L.F.J. Piper, ${ }^{1,3}$ A. Fedorov, ${ }^{4}$ T. Chen, ${ }^{5}$ T. D. Moustakas, ${ }^{5}$ and K.E. Smith ${ }^{1}$,* \\ ${ }^{1}$ Department of Physics, Boston University, Boston, MA 02215, USA \\ ${ }^{2}$ Department of Physcis, Gebze Institute Technology, Kocaeli, TR-41400 Turkey \\ ${ }^{3}$ Dept. of Phys, Appl. Phys. \& Astron., Binghamton University, Binghamton, NY 13902, USA \\ ${ }^{4}$ Advanced Light Source, Lawrence Berkeley National Laboratory, Berkeley, CA 94720, USA \\ ${ }^{5}$ Dept. of Electrical and Computer Engineering, Boston University, Boston, MA 02215, USA
}

\begin{abstract}
"We present angle resolved photoemission study of quantized electron accumulation subbands obtained from both clean and potassium deposited $\operatorname{InN}(000 \overline{1})$ surfaces. Shifting of the quantized accumulation states toward higher binding energies upon low energy $\mathrm{N}_{2}^{+}$ion bombardment or a small amount of potassium adsorption is explained by the modification of the In-adlayer induced surface states. $\mathrm{N}_{2}{ }^{+}$ion bombardment leads to a higher density of donor-type surface states by creating nitrogen vacancies near the surface. On the other hand, a small amount of $\mathrm{K}$ adsorbates initially donate their free electron to $\mathrm{InN}$ and result in more pronounced downward band bending. Eventually, further $\mathrm{K}$ adsorption leads to passivation of surface states and reduction of the surface electron accumulation. With the increase of the electron density, enhanced many-body interactions of electrons within the electron accumulation layer are observed."
\end{abstract}

\section{Introduction}


An electron accumulation layer in the conduction band is formed at clean surfaces or metal interfaces of nominally undoped InN. There has been strong interest in the study of electron accumulation formation on clean $\operatorname{InN}$ surfaces[1-8]; because the native electron accumulation on InN surfaces makes these materials very suitable for near infrared emission[9, 10], sensing devices[1, 11, 12], and high electron mobility transistors[13]. When the surface electron density is high enough, $\mathrm{E}_{\mathrm{F}}$ can be pinned above the $\mathrm{CBM}$. Quantization of the electron motion normal to the surface in the conduction band leads to discrete energy levels or electronic subbands. Thus, two-dimensional electron gas (2DEG) is formed at the surface due to strong downwards band bending. Several studies were performed to modify concentration of 2DEG on InN surface, such as oxidation of InN surface[14, 15], introduction of magnesium dopants[16], treatment of InN surface with $\mathrm{HCl}[6]$, and monolayer coverage of alkali metal[17]. However, all of these surface treatment procedures led to reduction of the surface electron accumulation. On the other hand, since 2DEG on III-V group semiconductors has an enormous potential with scientific and technological impact in future electronic devices, we have tried to increase the density of surface electrons by introducing either donor type defects created during surface cleaning by low energy ion bombardment or depositing a few percent of a monolayer of alkali metal on the surface.

In this paper, clean and potassium deposited $\operatorname{InN}(000 \overline{1})$ interfaces were studied via angleresolved photoemission spectroscopy (ARPES) proving that the 2D electron accumulation level can be controlled by either stoichiometric surface preparation or varying the amount of deposited potassium atoms on $\mathrm{InN}$ surface. We have previously investigated the surface electron accumulation of clean InN surface and observed that donor type defects or nitrogen vacancies are responsible for the pinning of $\mathrm{E}_{\mathrm{F}}$ up to $1 \mathrm{eV}$ above the $\mathrm{CBM}$ for $\mathrm{InN}$ cleaned by nitrogen ion 
sputtering[2]. Similar $\mathrm{E}_{\mathrm{F}}$ pinning is also observed by depositing very small amount of potassium on clean InN.

\section{Experimental Details}

Spectroscopic measurements were performed with $70 \mathrm{eV}$ photons with better than 35 meV energy resolution and angular resolution better than $0.5^{\circ}$ at ALS beam line 12.0.1 and 60 meV energy resolution and angular resolution better than $1^{\circ}$ at NSLS beam line U5UA, using Scienta analyzers with chamber pressures below $5 \times 10^{-11}$ torr. The InN films were grown on $c$ plane sapphire substrates by RF plasma-assisted molecular beam epitaxy[18]. The film was autodoped $n$-type with an average carrier concentration $5 \times 10^{19} \mathrm{~cm}^{3}$ and an average electron mobility $340 \mathrm{~cm}^{2} /$.s. The room temperature optical gap was approximately $0.77 \mathrm{eV}$. The sample was initially cleaned by annealing in UHV up to $400^{\circ} \mathrm{C}$. All surfaces exhibited sharp $1 \mathrm{x} 1$ LEED patterns with a low background. Then, clean samples were bombarded with $500 \mathrm{eV} \mathrm{N}_{2}^{+}$ ions and further annealed at $400^{\circ} \mathrm{C}$ in UHV. Potassium was deposited onto clean InN surfaces from a carefully outgassed SEAS getter source, without any pressure rise during deposition. Similar to the work of Betti et. al.[19], the amount of deposited K was calibrated by monitoring the change in the work function and Fermi level pinning. Variations in the work function was determined by applying a $-9 \mathrm{~V}$ potential to the sample. At saturation, the work function was reduced from the clean surface value by $2.6 \mathrm{eV}$. The accurate saturation coverage value was obtained from the variation of the energy difference between $\mathrm{E}_{\mathrm{F}}$ and the bottom of outer subband state as a function of work function shift, as seen in Fig. 1. A full surface coverage has been achieved by the point where no change in work function shift and band bending occurs.

Deposition of such a small amount of $\mathrm{K}$ did not have any noticeable effect on the surface electronic structure. During the photoemission measurements the angle of incidence of the 
photons was kept at $45^{\circ}$ and the electrons were detected form the normal emission direction. Binding energies are referenced to $E_{F}$, determined from a Au foil in contact with the sample. Although there is no observable difference in the core level energy positions at room temperature and lower temperatures, in order to eliminate the surface photovoltaic effect that may cause the similar results, we have performed the experiments at $100^{\circ} \mathrm{K}[20]$.

\section{Results and Discussion}

ARPES intensity map within $1.4 \mathrm{eV}$ of $\mathrm{E}_{\mathrm{F}}$ around the center of the Brillouin zone (BZ) along $\Gamma \rightarrow \mathrm{M}$, recorded with an incident photon energy of $70 \mathrm{eV}$, from clean $\mathrm{N}$-polar $\operatorname{InN}$ prepared by annealing in UHV to $400^{\circ} \mathrm{C}$, deposition of a small amount of potassium on clean InN, and two cycles of $500 \mathrm{eV} \mathrm{N}_{2}{ }^{+}$ion bombardment $(10 \mathrm{~min})$ and annealing in $\mathrm{UHV}$ to $400^{\circ} \mathrm{C}$ (10 min) are shown as 2D image plots along with the electron distribution curves (EDCs) in Figure 2(a), 2(b) and 2(c) respectively. The fundamental difference between 2(a) and 2(b) is the energy of the subband minima and correspondingly the Fermi wavevectors $\mathbf{k}_{\mathbf{F}}$ of the subbands. While the energy levels are localized at 0.4 and $0.15 \mathrm{eV}$ below $\mathrm{E}_{\mathrm{F}}$ for clean $\mathrm{InN}$, they are located at 0.55 and $0.23 \mathrm{eV}$ below $\mathrm{E}_{\mathrm{F}}$ after deposition of $0.02 \mathrm{ML}$ of potassium on clean $\mathrm{InN}$ surface. There is also another broad inner state very close to $\mathrm{E}_{\mathrm{F}}$ as shown by an arrow head in the profile, but it hard to determine the position from the image due to limits in the resolution of our system. However, three distinctive subbands were observed at $0.967 \mathrm{eV}, 0.61 \mathrm{eV}$ and $0.37 \mathrm{eV}$ below $\mathrm{E}_{\mathrm{F}}$ within the near surface region of $\mathrm{InN}$ surfaces prepared by two cycles of low energy $\mathrm{N}_{2}^{+}$ bombardment and UHV annealing. Similarly, two other broader inner bands observed from the

EDC. The effective masses $\left(\mathrm{m}^{*}\right)$ extracted from hyperbolic fits to the measured dispersions of Fig 1 yield $0.06 \mathrm{~m}_{\mathrm{e}}, 0.08 \mathrm{me}$ and $0.12 \mathrm{~m}_{\mathrm{e}}$ respectively [21]. While the first two values are consistent with the reported values of $\mathrm{m}^{*}$ which span between $0.044 \mathrm{~m}_{\mathrm{e}}$ and $0.093 \mathrm{~m}_{\mathrm{e}}[22]$; as the 
electron density in the conduction band is increased, effective mass of the electrons is doubled by decreasing the mobility of the electrons due to the interaction of the electrons with each other and defects. Another difference between three figures is the energy width of the subband peaks. The width of the subband states increases with potassium deposition or $\mathrm{N}_{2}{ }^{+}$ion sputtering due to scattering of the electrons from adsorbates or defects.

The relation between the amount of band bending and the spectral density of states can be evaluated from the energy levels in the conduction band. The increase in the energy levels and energy states is directly related to increase in the electron density at the bottom of the conduction band. The surface sheet densities extracted from $\mathbf{k}_{\mathbf{F}}$ are $1.9 \times 10^{13} \mathrm{~cm}^{-2}, 3.58 \times 10^{13} \mathrm{~cm}^{-2}$ and $7.7 \times 10^{13} \mathrm{~cm}^{-2}$ respectively for clean, potassium deposited, and mild energy ion bombarded $\mathrm{InN}$ surfaces. The data points can be well fitted by a parabola in narrow $\mathbf{k}_{\mathbf{F}}$ range of clean $\mathrm{InN}$, although, distortion from parabolic dispersion is observed for the potassium deposited and sputtered InN surfaces due to the enhanced interaction between valence and conduction band electrons with the increase in the conduction band electron density[23]. We previously investigated the effect of the strong electron-electron interaction onto the valence and conduction dispersions for $\mathrm{InN}$ after $\mathrm{N}_{2}{ }^{+}$ion sputtering and annealing $[2,23]$.

Figure 3 shows the photoemission EDCs as a function of potassium coverage around $\mathrm{E}_{\mathrm{F}}$. The discrete photoemission features observed at binding energies near $\mathrm{E}_{\mathrm{F}}$ are the electronic subbands in the conduction band of clean InN. The pinning of the $\mathrm{E}_{\mathrm{F}}$ above the CBM, due to donor type defects, leads to very strong band bending and quantized two dimensional electron subbands were formed on clean InN[6]. After deposition of 0.005ML of potassium, the energy levels of the subbands were shifted towards higher binding energy while there was no significant change in the structure of the energy levels. The binding energy of quantized electron 
accumulation states shifted to higher binding energy until deposition of about $0.02 \mathrm{ML}$ of potassium, and slight decrease in their intensity occurs due to scattering of electrons from $\mathrm{K}$ adsorbents. In addition, subbands in the conduction band slightly broaden and another state appeared as a shoulder to the inner band with further $\mathrm{K}$ deposition as seen in Fig. 3. The increased electron density in the conduction band due to further downward band bending allowed us to observe three energy levels after adsorption of a small amount of potassium atoms, although only two discrete states were observed for clean InN surface. The broadening of the quantum well states can be explained by scattering of the photoelectrons from the $\mathrm{K}$ ions or $2 \mathrm{D}$ clusters. To our knowledge there is no structural analysis was done on alkali metal/InN interface. However, $\mathrm{Cs}$ and $\mathrm{K}$ atoms adsorbed on III-V semiconductors at room temperature at very low coverage stay apart from each other or organize as zigzag chains; and at saturation coverage form 2D clusters [24-26]. Although the width of the subbands may depend on the atomic geometry of the metal adatoms, the electron density in the conduction band is determined by the depth of the potential well and does not depend on the distribution of the metal atoms on the surface[27]. With further deposition of potassium $(>0.02 \mathrm{ML})$, a shift towards lower binding energy and clear metallic Fermi edge was observed due to the metallic bond formed by K and In atoms on the surface. Unlike a typical semiconductor where Fermi level is inside the band bap, free electrons from metal atoms do not induce gap states, but they fill the energy levels in conduction band continuum. This leads to higher electron density and metallic Fermi edge.

Along with the increasing electron density in the conduction band, the variations in the intensity of subbands can be seen in the normal emission ARPES spectra normalized to the highest VB intensity covering the energy region of the upper valence band, band gap and the CBM in Figure 4. Solid and open triangles indicate the position of the top of the valence band 
and outer subband corresponding to the increase or decrease of energy levels due to adsorbed K amount. The top of the valence band was obtained by linear extrapolation of the leading edge. Here the top of the valence band is not actually the valence band maximum (VBM), because due to interactions between the subbands and valence band near the center of BZ, InN presents an inverted band structure and VBM is located about few degrees off normal[23]. At such a small coverage, not much effect was observed on the valence band structure (not shown here). However, when binding energies are referred to the $\mathrm{E}_{\mathrm{F}}$, it is clear that the valence-band edge shows $0.2 \mathrm{eV}$ shift to high binding energy due to the pinning of the $\mathrm{E}_{\mathrm{F}}$ into the conduction band until deposition of $0.02 \mathrm{ML}$ of potassium, and then moves towards lower binding energy with further K deposition. The In $4 \mathrm{~d}$ shallow core level energies were also measured with respect to Fermi level upon $\mathrm{K}$ adsorption, and similar downwards shift of the In $4 \mathrm{~d}$ states was observed as seen in the Fig. 4 inset. The maximum value of the downward band bending occurred at the same alkali metal coverage for which the highest electron accumulation was observed in the conduction band. The arrow heads on lower energy and higher energy indicate the satellite position

The origin of the electron accumulation in the conduction band of clean InN surface was investigated earlier and explained by Fermi level pinning due to the defects or donor type surface states induced by dangling In bonds at the surface[1, 2, 6, 28]. Both theoretical band structure calculations and experimental investigations of $\mathrm{InN}$ indicate that the stable structures of N-polar InN surfaces have been shown to be In-adlayer-terminated[6, 29, 30]. It is also predicted that downward band bending is caused by In dangling bond related occupied surface states which are located far above the CBM[31]. Further band bending upon alkali metal deposition or $\mathrm{N}_{2}^{+}$ion sputtering occurs due to modification of In-adlayer induced surface states. Betti et. al. 
investigated the modification of surface electron accumulation at InAs surface, and have shown that an accumulation layer forms after a small amount of $\mathrm{K}$ adsorption, however it decreases again with further K adsorption. Similar results were also reported for other alkali metals on IIIV semiconductors surfaces[32-34]. At low K coverages, electron transfer from potassium to underlying indium atoms forms K-In interface dipole and surface band bending becomes more pronounced. Upon further deposition, number of the $\mathrm{K}$ adatoms on the In-adlayer increases and it results in saturation of In dangling bond related surface states, which initially pin the Fermi level. Accordingly, subbands move towards their initial energy position of the clean surface and depletion of the electron concentration starts. The subband energy level shifts could also be explained by potassium induced gap states but there is no gap states observed within the band gap region of the whole BZ for such low alkali coverages. The reduction of surface downward band bending due to further $\mathrm{K}$ adsorbents is in agreement with earlier studies that found a reduction of the $\mathrm{InN}$ surface electron density by $1 \mathrm{ML}$ of $\mathrm{K}$ coverage of the surface. Eisenhardt et. al. measured a decrease of downward surface band bending by $0.2 \mathrm{eV}$ during $\mathrm{K}$ adsorption due to disappearance of In-adlayer induced surface states above conduction band[17].

The extreme band bending at sputtered and annealed InN surface is instead explained by the formation of donor-like defects during the sputtering (despite the low energies employed) most likely nitrogen vacancies localized within the near-surface region, consistent with earlier electron energy loss spectroscopy measurements[35]. During the preparation of clean $\mathrm{InN}$ surface by $\mathrm{N}_{2}{ }^{+}$ion sputtering and low temperature annealing, reformation of In and $\mathrm{N}$ atoms at the surface of InN occurs. $\mathrm{N}_{2}{ }^{+}$sputtering leads to a small amount increase in the number of metallic In atoms on the $\mathrm{N}_{2}^{+}$sputtered $\mathrm{InN}$ surface which exhibits larger downward band bending. A similar situation arises for the $\mathrm{N}_{2}{ }^{+}$sputtered GaN surface[36]. 


\section{Conclusion}

In conclusion, photoemission data showing the discrete electron accumulation states in the conduction band of clean and potassium deposited $\mathrm{InN}$ surfaces has been investigated. Surface charge density on InN can be enhanced by using mild ion bombardment and low temperature ( $400 \mathrm{C})$ annealing cycles. We also note that the $\mathrm{K}$ adsorption can be used as a method for controlled variation of the $2 \mathrm{D}$ electron density in the accumulation layer. A strong relationship between the charge density and potassium coverage was determined. At coverages as low as a few percent of a monolayer, the electron transfer from $\mathrm{K}$ atoms to $\mathrm{InN}$ creates downward band bending and surface electron density increases corresponding to this downward band binding. This identification suggests the potential to control the surface electronic properties of $\mathrm{InN}$ for applications in electronics and sensors.

\section{Acknowledgement}

This work was supported in part by the DOE under RF-06-PRD-001 and by the NSF under grant No. DMR-0311792.

\section{Figure Captions}

Figure 1. Fermi level pinning (the energy difference between $E_{F}$ and the bottom of outer subband state) vs work-function change, as deduced from the cutoff energy for the secondary electrons.

Figure 2. ARPES photocurrent intensity map of states within $1.4 \mathrm{eV}$ of $\mathrm{E}_{\mathrm{F}}$ along with EDCs for (a) clean InN surface obtained after annealed to $400^{\circ} \mathrm{C}$, (b) $\mathrm{K} / \mathrm{InN}$ surface obtained after deposition of $0.02 \mathrm{ML}$ of potassium on clean $\mathrm{InN}$ and (c) $\mathrm{InN}$ surface prepared by two cycles of $500 \mathrm{eV}$ Ar+ ion bombardment and annealing in $\mathrm{UHV}$ to $400^{\circ} \mathrm{C}$. The momentum direction is 
along $\Gamma \rightarrow \mathrm{M}$ in the surface plane.

Figure 3. Series of EDCs for $\mathrm{K} / \mathrm{InN}(000 \overline{1})$ interface showing the conduction band minimum region for different potassium evaporation time taken at $h v=70 \mathrm{eV}$ after the sample is annealed to $400^{\circ} \mathrm{C}$ in UHV. Solid and open triangles indicate the position of the top of valence band and outer subband for each spectra, respectively.

Figure 4. Photoemission spectra of $\mathrm{K} / \mathrm{InN}(000 \overline{1})$ interface showing the upper valence band, band gap and the conduction band minimum region for different coverages of potassium. Inset shows the In $4 \mathrm{~d}$ core level normalized spectra of the $\mathrm{K} / \mathrm{InN}$ interface at different $\mathrm{K}$ coverages.

\section{References}

*ksmith@bu.edu

1. Mahboob, I., et al., Origin of electron accumulation at wurtzite InN surfaces. Physical Review B, 2004. 69(20): p. 201307.

2. Colakerol, L., et al., Quantized electron accumulation states in indium nitride studied by angle-resolved photoemission spectroscopy. Physical Review Letters, 2006. 97(23): p. 237601

3. Segura-Ruiz, J., et al., Optical studies of MBE-grown InN nanocolumns: Evidence of surface electron accumulation. Physical Review B, 2009. 79(11): p. 115305.

4. Cho, Y.J., M. Ramsteiner, and O. Brandt, Observation of the electron-accumulation layer at the surface of InN by cross-sectional micro-Raman spectroscopy. Applied Physics Letters, 2013. 102(7): p. 072101.

5. Zhao, S., et al., Tuning the Surface Charge Properties of Epitaxial InN Nanowires. Nano Letters, 2012. 12(6): p. 2877-2882.

6. Kuo, C.T., et al., Is electron accumulation universal at InN polar surfaces? Applied Physics Letters, 2011. 98(5): p. 052101.

7. Darakchieva, V., et al., Free electron behavior in InN: On the role of dislocations and surface electron accumulation. Applied Physics Letters, 2009. 94(2): p. 022109.

8. Bhatta, R.P., et al., Carrier concentration and surface electron accumulation in indium nitride layers grown by high pressure chemical vapor deposition. Journal of Vacuum Science \& Technology A, 2007. 25(4): p. 967-970.

9. Shen, C.H., et al., Near-infrared photoluminescence from vertical InN nanorod arrays grown on silicon: Effects of surface electron accumulation layer. Applied Physics Letters, 2006. 88(25): p. 253104-3.

10. Stoica, T., et al., Photoluminescence and Intrinsic Properties of MBE-Grown InN Nanowires. Nano Letters, 2006. 6(7): p. 1541-1547.

11. Kryliouk, O., et al., Pt-coated InN nanorods for selective detection of hydrogen at room temperature. Journal of Vacuum Science \& Technology B: Microelectronics and Nanometer Structures, 2005. 23(5): p. 1891-1894.

12. Lu, Y.-S., et al., InN-based anion selective sensors in aqueous solutions. Applied Physics Letters, 2007. 91(20): p. 202109-3.

13. Kuzmik, J., N-Polarity InN/GaN/InAlN High-Electron-Mobility Transistors. Applied Physics Express, 2012. 5(4): p. 044101.

14. Cimalla, V., et al., Reduced surface electron accumulation at InN films by ozone induced oxidation. Applied Physics Letters, 2007. 90(15): p. 152106. 
15. Lebedev, V., et al., Effect of surface oxidation on electron transport in InN thin films. Journal of Applied Physics, 2007. 101(12): p. 123705.

16. Linhart, W.M., et al., Giant Reduction of InN Surface Electron Accumulation: Compensation of Surface Donors by Mg Dopants. Physical Review Letters, 2012. 109(24): p. 247605.

17. Eisenhardt, A., et al., Reduction of electron accumulation at InN(0001) surfaces via saturation of surface states by potassium and oxygen as donor- or acceptor-type adsorbates. Journal of Applied Physics, 2014. 115(4): p. 043716.

18. Chen, T.C.P., et al., Growth of InN films by RF plasma-assisted MBE and cluster beam epitaxy. Journal of Crystal Growth, 2006. 288(2): p. 254-260.

19. Betti, M.G., et al., Density of states of a two-dimensional electron gas at semiconductor surfaces. Physical Review B, 2001. 63(15): p. 155315.

20. Hecht, M.H., Photovoltaic Effects in Photoemission-Studies of Schottky-Barrier Formation. Journal of Vacuum Science \& Technology B, 1990. 8(4): p. 1018-1024.

21. Millot, M., et al., Determination of effective mass in InN by high-field oscillatory magnetoabsorption spectroscopy. Physical Review B, 2011. 83(12): p. 125204.

22. Goiran, M., et al., Electron cyclotron effective mass in indium nitride. Applied Physics Letters, 2010. 96(5): p. 052117.

23. Colakerol, L., et al., Observation of an inverted band structure near the surface of InN. Epl, 2008. 83(4): p. 47003.

24. Aristov, V.Y., et al., Giant Band Bending and Interface Formation of Cs/Inas(110) at Room-Temperature. Journal De Physique Iv, 1994. 4(C9): p. 217-220.

25. Gavioli, L., et al., Self-assembling of potassium nanostructures on InAs(110) surface. Surface Science, 2003. 532: p. 666-670.

26. Mariani, C., A high-resolution photoemission study of confined metal systems on InAs(110). Surface Science, 2000. 454: p. 417-427.

27. Betti, M.G., et al., Density of states of a two-dimensional electron gas measured by high-resolution photoelectron spectroscopy. Solid State Communications, 1999. 110(12): p. 661-666.

28. Nagata, T., et al., Surface structure and chemical states of a-plane and c-plane InN films. Applied Physics Letters, 2009. 95(13): p. 132104.

29. Gan, C.K. and D.J. Srolovitz, First-principles study of wurtzite InN (0001) and (000(1)over-bar) surfaces. Physical Review B, 2006. 74(11): p. 115319.

30. Wu, C.L., et al., Absence of Fermi-level pinning at cleaved nonpolar InN surfaces. Physical Review Letters, 2008. 101(10): p. 106803

31. Van de Walle, C.G. and D. Segev, Microscopic origins of surface states on nitride surfaces. Journal of Applied Physics, 2007. 101(8): p. 081704.

32. Aristov, V.Y., et al., Sb or Cs covered InAs(110) surfaces: Moving E(F) into conduction band and quantized 2D electron channel. Applied Surface Science, 1996. 104: p. 73-78.

33. Aristov, V.Y., et al., Direct measurement of quantum-state dispersion in an accumulation layer at a semiconductor surface. Physical Review B, 1999. 60(11): p. 7752-7755.

34. Schirm, K.M., et al., Schottky-Barrier and Interface Formation of Cs/Gasb(110) and Rb/Gasb(110) at Room-Temperature. Physical Review B, 1994. 49(8): p. 5490-5496.

35. Piper, L.F.J., et al., InN: Fermi level stabilization by low-energy ion bombardment. Physica Status Solidi C - Current Topics in Solid State Physics, Vol 3, No 6, 2006. 3(6): p. 1841-1845.

36. Lai, Y.H., et al., Sputtering and etching of GaN surfaces. Journal of Physical Chemistry B, 2001. 105(41): p. $10029-10036$. 


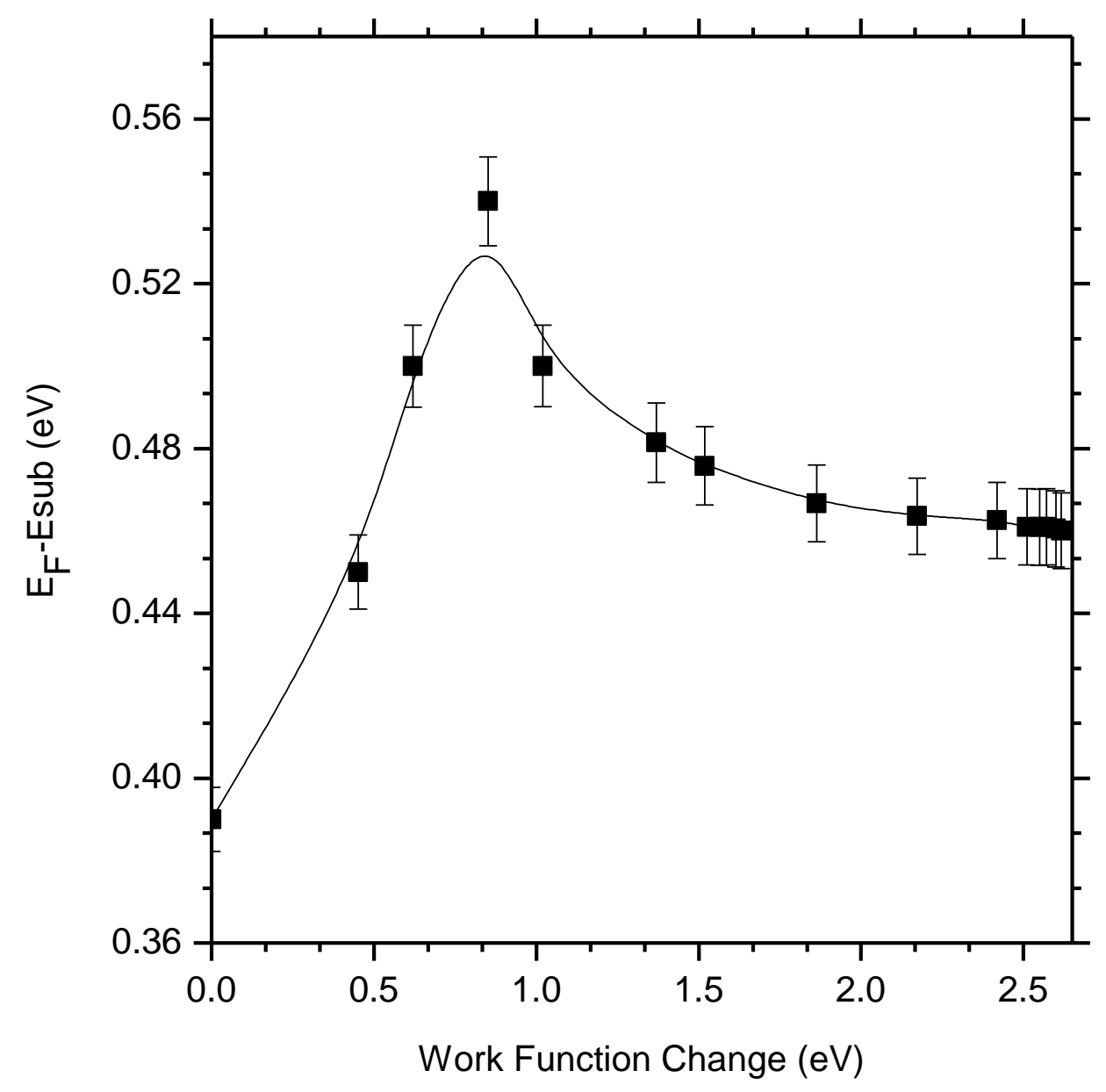

Figure 1. Fermi level pinning (the energy difference between $E_{F}$ and the bottom of outer subband state) vs work-function change, as deduced from the cutoff energy for the secondary electrons. 

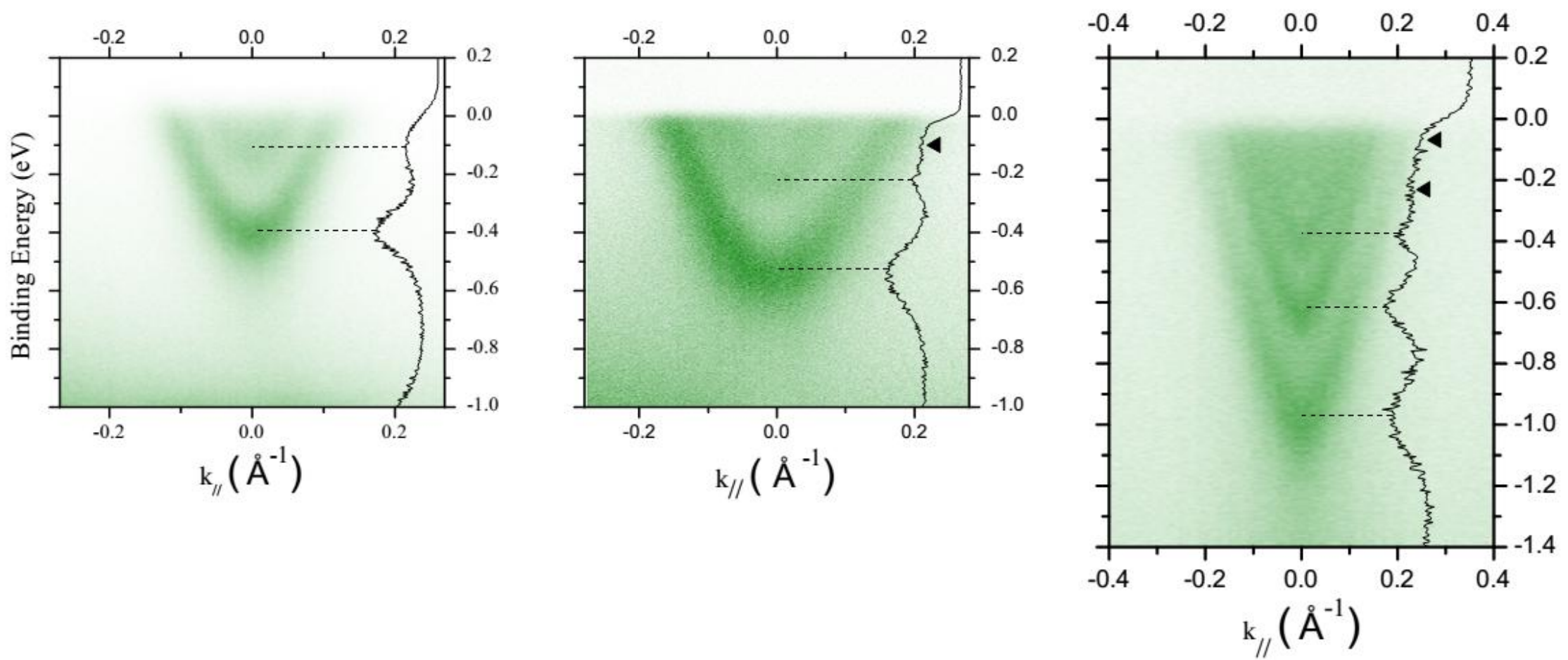

Figure 2. ARPES photocurrent intensity map of states within $1.4 \mathrm{eV}$ of $\mathrm{E}_{\mathrm{F}}$ along with EDCs for (a) clean InN surface obtained after annealed to $400^{\circ} \mathrm{C}$, (b) $\mathrm{K} / \mathrm{InN}$ surface obtained after deposition of $0.02 \mathrm{ML}$ of potassium on clean $\operatorname{lnN}$ and (c) InN surface prepared by two cycles of $500 \mathrm{eV} \mathrm{Ar}+$ ion bombardment and annealing in $\mathrm{UHV}$ to $400^{\circ} \mathrm{C}$. The momentum direction is along $\Gamma \rightarrow \mathrm{M}$ in the surface plane. 


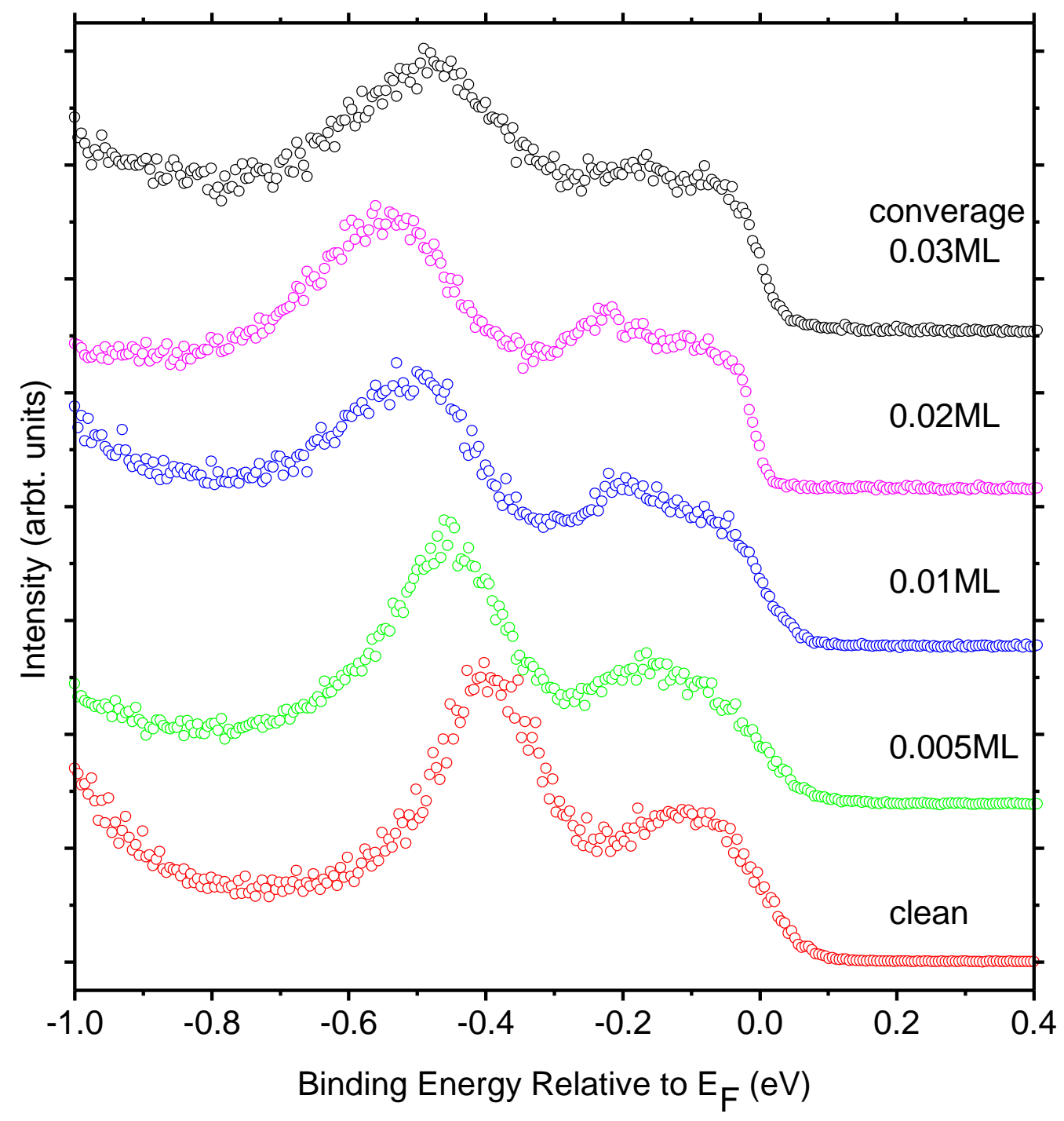

Figure 3. Series of EDCs for $\mathrm{K} / \mathrm{lnN}(000-1)$ interface showing the conduction band minimum region for different potassium evaporation time taken at $h v=70 \mathrm{eV}$ after the sample is annealed to $400^{\circ} \mathrm{C}$ in UHV. Solid and open triangles indicate the position of the top of valence band and outer subband for each spectra, respectively. 


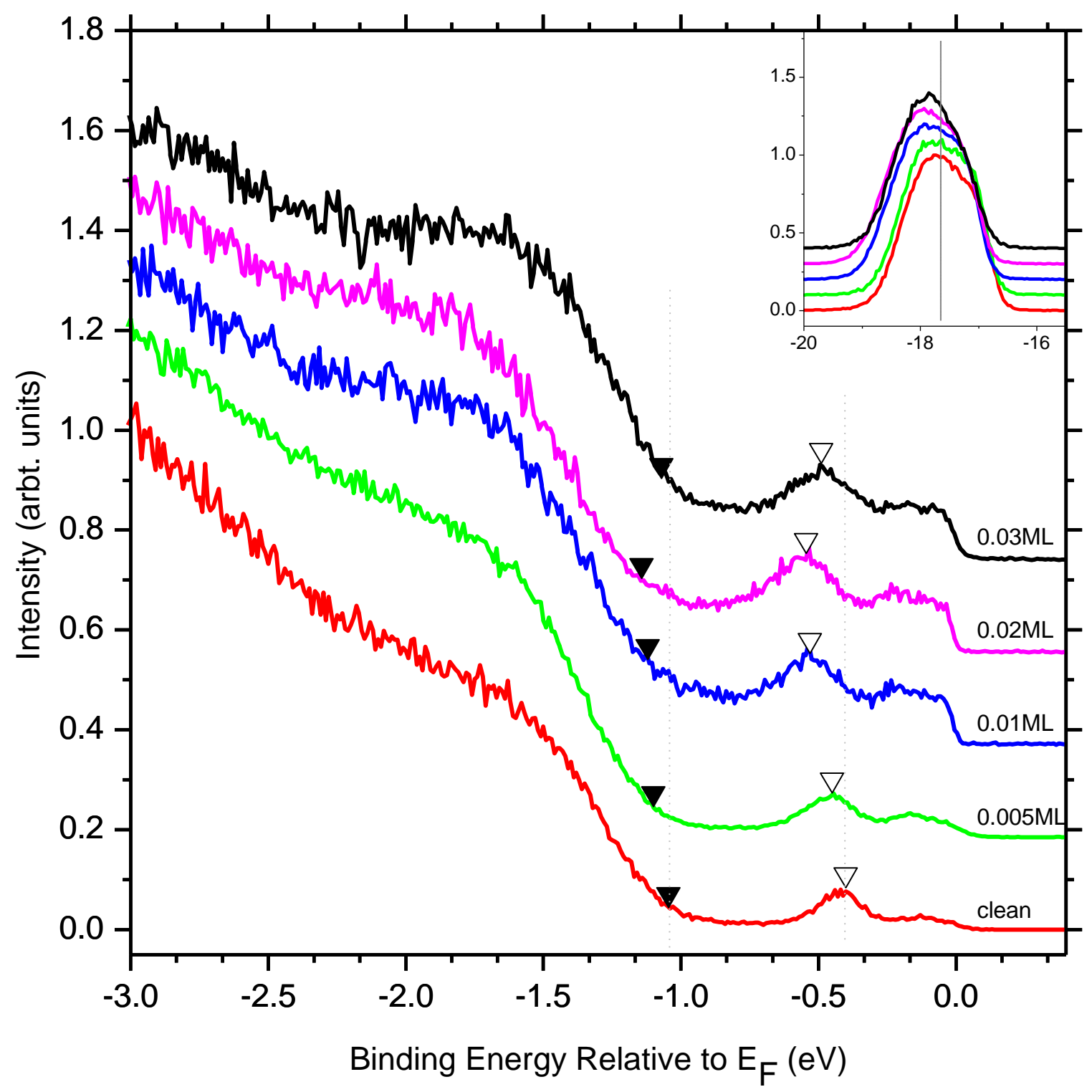

Figure 4. Photoemission spectra of $\mathrm{K} / \mathrm{lnN}(000-1)$ interface showing the upper valence band, band gap and the conduction band minimum region for different coverages of potassium. Inset shows the In $4 d$ core level normalized spectra of the $\mathrm{K} / \mathrm{InN}$ interface at different $\mathrm{K}$ coverages. 